\title{
Analysis of accelerations in turbulence based on generalized statistics
}

\author{
T.Arimitsu ${ }^{1}$, N.Arimitsu ${ }^{2 \dagger}$ \\ 1 Institute of Physics, University of Tsukuba, Ibaraki 305-8571, Japan \\ 2 Graduate School of EIS, Yokohama Nat'l. University, \\ Kanagawa 240-8501, Japan
}

Received October 14, 2002

An analytical expression of probability density function (PDF) of accelerations in turbulence is derived with the help of the statistics based on generalized entropy (the Tsallis entropy or the Rényi entropy). It is revealed that the derived PDF explains the one obtained by Bodenschatz et al. in the measurement of fluid particle accelerations in fully developed turbulence at $R_{\lambda}=970$.

Key words: multifractal analysis, fully developed turbulence, PDF of fluid particle accelerations, Rényi entropy, Tsallis entropy

PACS: 47.27.-i, 47.53. $+n, 47.52 .+j, 05.90 .+m$

The multifractal analysis of turbulence by the statistics based on the generalized entropy of Rényi's or of Tsallis' has been developed by the present authors [1-9]. Similarly to the usual thermodynamic entropy, the Rényi entropy [10] is of an extensive character, whereas the Tsallis entropy [11-13] is non-extensive. The multifractal analysis belongs to the line of study based on a kind of ensemble theoretical approaches that, starting from the log-normal model [14-16], continues with the $\beta$-model [17], the p-model $[18,19]$, the 3D binomial Cantor set model [20] and so on. After a rather preliminary investigation of the p-model [1], we developed further to derive the analytical expression for the scaling exponents of velocity structure function [2-5], and to determine the probability density function (PDF) of velocity fluctuations [5-8] and of velocity derivative [9] by a self-consistent statistical mechanical approach.

In this paper, we will derive the formula for the PDF of the accelerations of a fluid particle in fully developed turbulence by means of the multifractal analysis. With the theoretical PDF, we will analyze the PDF of accelerations at $R_{\lambda}=970$ (the Taylor microscale Reynolds number) obtained in the Lagrangian measurement

*E-mail: arimitsu@cm.ph.tsukuba.ac.jp

$\dagger$ E-mail: arimitsu@ynu.ac.jp 
of particle accelerations that was realized by Bodenschatz and co-workers [21,22] by raising dramatically the spatial and temporal measurement resolutions with the help of the silicon strip detectors.

We assume that the turbulent flow, satisfying the Navier-Stokes equation

$$
\partial \vec{u} / \partial t+(\vec{u} \cdot \vec{\nabla}) \vec{u}=-\vec{\nabla}(p / \rho)+\nu \nabla^{2} \vec{u}
$$

of an incompressible fluid, consists of a cascade of eddies with different sizes $\ell_{n}=$ $\delta_{n} \ell_{0}$, where $\delta_{n}=2^{-n}(n=0,1,2, \cdots)$. The quantities $\rho, p$ and $\nu$ represent, respectively, the mass density, the pressure and the kinematic viscosity. The acceleration $\vec{a}$ of a fluid particle is given by the substantive time derivative of the velocity: $\overrightarrow{\mathrm{a}}=\partial \vec{u} / \partial t+(\vec{u} \cdot \vec{\nabla}) \vec{u}$. At each step of the cascade, say at the $n$th step, eddies break up into two pieces producing an energy cascade with the energy-transfer rate $\epsilon_{n}$ that represents the rate of transfer of energy per unit mass from eddies of size $\ell_{n}$ to those of size $\ell_{n+1}$ (the energy cascade model). The Reynolds number Re of the system is given by $\operatorname{Re}=\delta u_{0} \ell_{0} / \nu=\left(\ell_{0} / \eta\right)^{4 / 3}$ with the Kolmogorov scale [23] $\eta=\left(\nu^{3} / \epsilon\right)^{1 / 4}$, where $\epsilon\left(=\epsilon_{0}\right)$ is the energy input rate to the largest eddies with size $\ell_{0} \cdot{ }^{1}$ Introducing the pressure (divided by the mass density) difference $\delta p_{n}$ at two points separated by the distance $\ell_{n}$, i.e., $\delta p_{n}=\left|p / \rho\left(\bullet+\ell_{n}\right)-p / \rho(\bullet)\right|$, and the acceleration $\mathrm{a}_{n}=\delta p_{n} / \ell_{n}$ belonging to the $n$th step in the energy cascade, one can estimate accelerations by $|\vec{a}|=\lim _{n \rightarrow \infty} \mathrm{a}_{n}$. For a high Reynolds number Re $\gg 1$, or for the situation where the effects of the kinematic viscosity $\nu$ can be neglected compared with those of the turbulent viscosity, the Navier-Stokes equation (1) is invariant under the scale transformation [24,19]: $\vec{r} \rightarrow \lambda \vec{r}, \vec{u} \rightarrow \lambda^{\alpha / 3} \vec{u}, t \rightarrow \lambda^{1-\alpha / 3} t$ and $(p / \rho) \rightarrow \lambda^{2 \alpha / 3}(p / \rho)$. The exponent $\alpha$ is an arbitrary real quantity which specifies the degree of singularity in the acceleration for $\alpha<1.5$, i.e., $\lim _{n \rightarrow \infty} \mathrm{a}_{n}=\lim _{\ell_{n} \rightarrow 0} \delta p_{n} / \ell_{n} \sim \lim _{\ell_{n} \rightarrow 0} \ell_{n}^{(2 \alpha / 3)-1} \rightarrow \infty$ which can be seen with the relation $\delta p_{n} / \delta p_{0}=\left(\ell_{n} / \ell_{0}\right)^{2 \alpha / 3}$.

The multifractal analysis rests on the assumption that the distribution of the exponent $\alpha$ is multifractal, and that the probability $P^{(n)}(\alpha) \mathrm{d} \alpha$ to find, at a point in physical space, an eddy of size $\ell_{n}$ having a value of the degree of singularity in the range $\alpha \sim \alpha+\mathrm{d} \alpha$ is given by [2-5]

$$
P^{(n)}(\alpha) \propto\left[1-\left(\alpha-\alpha_{0}\right)^{2} /(\Delta \alpha)^{2}\right]^{n /(1-q)}
$$

with $(\Delta \alpha)^{2}=2 X /[(1-q) \ln 2]$. The range of $\alpha$ is $\alpha_{\min } \leqslant \alpha \leqslant \alpha_{\max }$ with $\alpha_{\min }=$ $\alpha_{0}-\Delta \alpha$ and $\alpha_{\max }=\alpha_{0}+\Delta \alpha$. Here, we assume that the distribution function at the $n$th multifractal depth has the structure $P^{(n)}(\alpha) \propto\left[P^{(1)}(\alpha)\right]^{n}$. This is consistent with the relation $[19,5] P^{(n)}(\alpha) \propto \delta_{n}^{1-f(\alpha)}$ that is a manifestation of scale inveriance and reveals how densely each singularity, labeled by $\alpha$, fills the physical space. Within the present model, the multifractal spectrum $f(\alpha)$ is given by $[2-5]$

$$
f(\alpha)=1+(1-q)^{-1} \log _{2}\left[1-\left(\alpha-\alpha_{0}\right)^{2} /(\Delta \alpha)^{2}\right]
$$

\footnotetext{
${ }^{1}$ The velocity fluctuation $\delta u_{n}$ is defined by $\delta u_{n}=\left|u\left(\bullet+\ell_{n}\right)-u(\bullet)\right|$ where $u$ represents a component of the velocity field $\vec{u}$.
} 
To make the paper self-contained, we put here its brief derivation. The distribution function (2) is derived by taking an extremum of the generalized entropy, the Rényi entropy [10] $S_{q}^{\mathrm{R}}\left[P^{(1)}(\alpha)\right]=(1-q)^{-1} \ln \int \mathrm{d} \alpha P^{(1)}(\alpha)^{q}$ or the Tsallis entropy $[11-13] S_{q}^{\mathrm{T}}\left[P^{(1)}(\alpha)\right]=(1-q)^{-1}\left(\int \mathrm{d} \alpha P^{(1)}(\alpha)^{q}-1\right)$, under the two constraints, i.e., the normalization of distribution function: $\int \mathrm{d} \alpha P^{(1)}(\alpha)=$ const and the $q$-variance being kept constant as a known quantity: $\sigma_{q}^{2}=\left(\int \mathrm{d} \alpha P^{(1)}(\alpha)^{q}(\alpha-\right.$ $\left.\left.\alpha_{0}\right)^{2}\right) / \int \mathrm{d} \alpha P^{(1)}(\alpha)^{q}$. In spite of the different characteristics of these entropies, the distribution function giving their extremum has the common structure (2). The dependence of the parameters $\alpha_{0}, X$ and $q$ on the intermittency exponent $\mu$ is determined, self-consistently, with the help of three independent equations, i.e., the energy conservation: $\left\langle\epsilon_{n}\right\rangle=\epsilon$, the definition of the intermittency exponent $\mu:\left\langle\epsilon_{n}^{2}\right\rangle=\epsilon^{2} \delta_{n}^{-\mu}$, and the scaling relation: ${ }^{2} 1 /(1-q)=1 / \alpha_{-}-1 / \alpha_{+}$with $\alpha_{ \pm}$satisfying $f\left(\alpha_{ \pm}\right)=0$. The average $\langle\cdots\rangle$ is taken with $P^{(n)}(\alpha)$. For the region where the value of $\mu$ is usually observed, i.e., $0.13 \leqslant \mu \leqslant 0.40$, the three self-consistent equations are solved to give the approximate equations [8]: $\alpha_{0}=0.9989+0.5814 \mu, X=-2.848 \cdot 10^{-3}+1.198 \mu$ and $q=-1.507+20.58 \mu-97.11 \mu^{2}+260.4 \mu^{3}-365.4 \mu^{4}+208.3 \mu^{5}$.

Since there are two mechanisms in a turbulent flow to rule its evolution, i.e., the one controlled by the kinematic viscosity that takes care of thermal fluctuations, and the other by the turbulent viscosity that is responsible for intermittent fluctuations related to the singularities in acceleration, it may be reasonable to assume that the probability $\Lambda^{(n)}\left(y_{n}\right) \mathrm{d} y_{n}$ to find the scaled pressure fluctuations $\left|y_{n}\right|=\delta p_{n} / \delta p_{0}$ in the range $y_{n} \sim y_{n}+\mathrm{d} y_{n}$ has two independent origins:

$$
\Lambda^{(n)}\left(y_{n}\right) \mathrm{d} y_{n}=\Lambda_{\mathrm{S}}^{(n)}\left(\left|y_{n}\right|\right) \mathrm{d} y_{n}+\Delta \Lambda^{(n)}\left(y_{n}\right) \mathrm{d} y_{n} .
$$

The singular part $\Lambda_{\mathrm{S}}^{(n)}\left(\left|y_{n}\right|\right)$ of the PDF stemmed from multifractal distribution of the singularities, and the correction part $\Delta \Lambda^{(n)}\left(y_{n}\right)$ from the thermal dissipation and/or the measurement error. ${ }^{3}$ The former is derived through $\Lambda_{\mathrm{S}}^{(n)}\left(\left|y_{n}\right|\right) \mathrm{d} y_{n} \propto$ $P^{(n)}(\alpha) \mathrm{d} \alpha$ with the transformation of the variables: $\left|y_{n}\right|=\delta_{n}^{2 \alpha / 3}$. The $m$ th moments of the pressure fluctuations are given by

$$
\left\langle\left\langle\left|y_{n}\right|^{m}\right\rangle\right\rangle \equiv \int_{-\infty}^{\infty} \mathrm{d} y_{n}\left|y_{n}\right|^{m} \Lambda^{(n)}\left(y_{n}\right)=2 \tilde{\gamma}_{m}^{(n)}+\left(1-2 \tilde{\gamma}_{0}^{(n)}\right) a_{2 m} \delta_{n}^{\zeta_{2 m}}
$$

with $a_{3 \bar{q}}=\left\{2 /\left[\sqrt{C_{\bar{q}}}\left(1+\sqrt{C_{\bar{q}}}\right)\right]\right\}^{1 / 2}, C_{\bar{q}}=1+2 \bar{q}^{2}(1-q) X \ln 2$ and

$$
2 \tilde{\gamma}_{m}^{(n)}=\int_{-\infty}^{\infty} \mathrm{d} y_{n}\left|y_{n}\right|^{m} \Delta \Lambda^{(n)}\left(y_{n}\right)
$$

\footnotetext{
${ }^{2}$ The scaling relation is a generalization of the one derived first in $[25,26]$ to the case where the multifractal spectrum has negative values [1].

${ }^{3}$ Needless to say that each term in (4) is a multiple of two PDF's, i.e., the PDF for one of the two independent origins to realize and the conditional PDF for a value $y_{n}$ in the range $y_{n} \sim y_{n}+\mathrm{d} y_{n}$ to come out. This is, of course, in a generalized sense in which the second correction term may weaken the first singular contribution.
} 


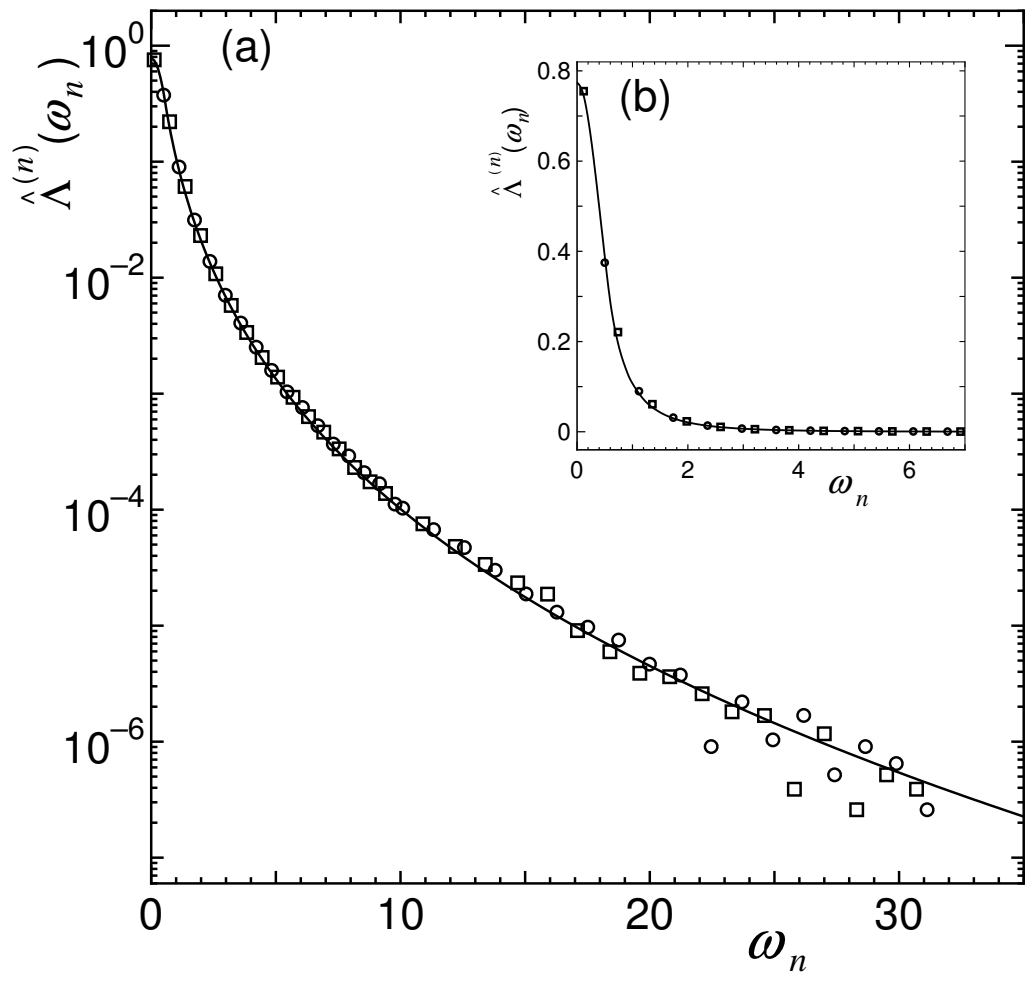

Figure 1. PDF of accelerations plotted on (a) log and (b) linear scale. Comparison between the experimentally measured PDF of fluid particle accelerations by Bodenschatz et al. at $R_{\lambda}=970(\mathrm{Re}=62700)$ and the present theoretical PDF $\hat{\Lambda}^{(n)}\left(\omega_{n}\right)$. Open squares are the experimental data points on the left hand side of the PDF, whereas open circles are those on the right hand side. Solid lines represent the curves given by the present theory (8) with $\mu=0.259(q=0.431)$ and $n=16.4$.

We used the normalization: $\langle\langle 1\rangle\rangle=1$. The quantity

$$
\zeta_{m}=\frac{\alpha_{0} m}{3}-\frac{2 X m^{2}}{9\left(1+\sqrt{C_{m / 3}}\right)}-\frac{1}{1-q}\left[1-\log _{2}\left(1+\sqrt{C_{m / 3}}\right)\right]
$$

is the so-called scaling exponent of velocity structure function, whose expression was originally derived by the present authors [2-5]. The formula quite well explains the experimental data $[2-6,8]$. Note that the formula is independent of the length $\ell_{n}$, and, therefore, independent of $n$.

Let us derive the PDF $\hat{\Lambda}^{(n)}\left(\omega_{n}\right)$ of the accelerations by dividing it into two parts with respect to $\omega_{n}$, i.e.,

$$
\hat{\Lambda}^{(n)}\left(\omega_{n}\right)= \begin{cases}\hat{\Lambda}_{<\dagger}^{(n)}\left(\omega_{n}\right) & \text { for }\left|\omega_{n}\right| \leqslant \omega_{n}^{\dagger} \\ \hat{\Lambda}_{\dagger<}^{(n)}\left(\omega_{n}\right) & \text { for } \omega_{n}^{\dagger} \leqslant\left|\omega_{n}\right| \leqslant \omega_{n}^{\max }\end{cases}
$$

where the scaled variable $\omega_{n}$ is defined by $\left|\omega_{n}\right|=\mathrm{a}_{n} /\left\langle\left\langle\mathrm{a}_{n}^{2}\right\rangle\right\rangle^{1 / 2}=\bar{\omega}_{n} \delta_{n}^{2 \alpha / 3-\zeta_{4} / 2}$ with $\bar{\omega}_{n}=\left[2 \tilde{\gamma}_{2}^{(n)} \delta_{n}^{-\zeta_{4}}+\left(1-2 \tilde{\gamma}_{0}^{(n)}\right) a_{4}\right]^{-1 / 2}$, and $\omega_{n}^{\max }=\bar{\omega}_{n} \delta_{n}^{2 \alpha_{\min } / 3-\zeta_{4} / 2}$. Assuming that, for 
smaller accelerations $\left|\omega_{n}\right| \leqslant \omega_{n}^{\dagger}$, the contribution to the PDF comes, mainly, from thermal fluctuations related to the kinematic viscosity or from the measurement error, we take for the PDF $\hat{\Lambda}_{<\dagger}^{(n)}\left(\omega_{n}\right) \omega_{n}=\Lambda_{\mathrm{S}}^{(n)}\left(y_{n}\right) \mathrm{d} y_{n}+\Delta \Lambda^{(n)}\left(y_{n}\right) \mathrm{d} y_{n}$ a Gaussian function, i.e.,

$$
\hat{\Lambda}_{<\dagger}^{(n)}\left(\omega_{n}\right)=\tilde{\Lambda}_{\mathrm{S}}^{(n)} \exp \left\{-\frac{1}{2}\left[1+\frac{3}{2} f^{\prime}\left(\alpha^{\dagger}\right)\right]\left[\left(\omega_{n} / \omega_{n}^{\dagger}\right)^{2}-1\right]\right\}
$$

with $\tilde{\Lambda}_{\mathrm{S}}^{(n)}=3\left(1-2 \tilde{\gamma}_{0}^{(n)}\right) /\left(4 \bar{\omega}_{n} \sqrt{2 \pi X\left|\ln \delta_{n}\right|}\right)$. On the other hand, we assume that the main contribution to $\hat{\Lambda}_{+<}^{(n)}\left(\omega_{n}\right)$ may come from the multifractal distribution of singularities related to the turbulent viscosity, i.e., $\hat{\Lambda}_{\dagger<}^{(n)}\left(\omega_{n}\right) \mathrm{d} \omega_{n}=\Lambda_{\mathrm{S}}^{(n)}\left(\left|y_{n}\right|\right) \mathrm{d} y_{n}$ :

$$
\hat{\Lambda}_{\dagger<}^{(n)}\left(\omega_{n}\right)=\tilde{\Lambda}_{\mathrm{S}}^{(n)} \frac{\bar{\omega}_{n}}{\left|\omega_{n}\right|}\left[1-\frac{1-q}{n} \frac{\left(3 \ln \left|\omega_{n} / \omega_{n, 0}\right|\right)^{2}}{8 X\left|\ln \delta_{n}\right|}\right]^{n /(1-q)}
$$

with $\left|\omega_{n, 0}\right|=\bar{\omega}_{n} \delta_{n}^{2 \alpha_{0} / 3-\zeta_{4} / 2}$. The point $\omega_{n}^{\dagger}$ was defined by $\omega_{n}^{\dagger}=\bar{\omega}_{n} \delta_{n}^{2 \alpha^{\dagger} / 3-\zeta_{4} / 2}$ with $\alpha^{\dagger}$ being the smaller solution of $\zeta_{4} / 2-2 \alpha / 3+1-f(\alpha)=0$, at which $\hat{\Lambda}^{(n)}\left(\omega_{n}^{\dagger}\right)$ has the least $n$-dependence for large $n$. Here, $\hat{\Lambda}_{<\dagger}^{(n)}\left(\omega_{n}\right)$ and $\hat{\Lambda}_{\dagger<}^{(n)}\left(\omega_{n}\right)$ were connected at $\omega_{n}^{\dagger}$ under the condition that they should have the same value and the same slope there. The specific form of Gaussian function (9) comes out through the connection.

With the expression (9), we can obtain $\Delta \Lambda^{(n)}\left(y_{n}\right)$, and have the formula to evaluate $\tilde{\gamma}_{m}^{(n)}$ in the form

$$
2 \tilde{\gamma}_{m}^{(n)}=\left(K_{m}^{(n)}-L_{m}^{(n)}\right) /\left(1+K_{0}^{(n)}-L_{0}^{(n)}\right)
$$

where

$$
\begin{aligned}
K_{m}^{(n)} & =\frac{3 \delta_{n}^{2(m+1) \alpha^{\dagger} / 3-\zeta_{4} / 2}}{2 \sqrt{2 \pi X\left|\ln \delta_{n}\right|}} \int_{0}^{1} \mathrm{~d} z z^{m} \exp \left\{-\frac{1}{2}\left[1+\frac{3}{2} f^{\prime}\left(\alpha^{\dagger}\right)\right]\left(z^{2}-1\right)\right\} \\
L_{m}^{(n)} & =\frac{3 \delta_{n}^{2 m \alpha^{\dagger} / 3}}{2 \sqrt{2 \pi X\left|\ln \delta_{n}\right|}} \int_{z_{\min }}^{1} \mathrm{~d} z z^{m-1}\left[1-\frac{1-q}{n} \frac{\left(3 \ln \left|z / z_{0}^{\dagger}\right|\right)^{2}}{8 X\left|\ln \delta_{n}\right|}\right]^{n /(1-q)}
\end{aligned}
$$

with $z_{\min }=\omega_{\min } / \omega_{n}^{\dagger}=\delta_{n}^{2\left(\alpha_{\max }-\alpha^{\dagger}\right) / 3}$ and $z_{0}^{\dagger}=\omega_{n, 0} / \omega_{n}^{\dagger}=\delta_{n}^{2\left(\alpha_{0}-\alpha^{\dagger}\right) / 3}$. Now, the PDF of fluid particle accelerations (8) is determined by the intermittency exponent $\mu$ and the number $n$ of steps in the energy cascade which gives the eddy size $\ell_{n}$.

The comparison between the present PDF of accelerations and that measured in the experiment $[21,22]$ at $R_{\lambda}=970$ is plotted in figure 1 on log and linear scale. The intermittency exponent $\mu=0.259$ and the number $n=16.4$ of steps in the cascade are extracted by the method of least squares with respect to the logarithm of PDF's as the best fit of our theoretical formulae (8) to the observed values of the PDF $[21,22]$ by discarding those points whose values are less than $\sim 2 \cdot 10^{-6}$ since they 
Table 1. The values of the scaling exponents derived by the present theory for the acceleration is also listed with the corresponding values for $q, \alpha_{0}$ and $X$.

\begin{tabular}{cc|cc|cc|cc}
$m$ & $\zeta_{m}$ & $m$ & $\zeta_{m}$ & $m$ & $\zeta_{m}$ & $m$ & $\zeta_{m}$ \\
\hline 1 & 0.3661 & 6 & 1.741 & 11 & 2.599 & 16 & 3.175 \\
2 & 0.6989 & 7 & 1.945 & 12 & 2.731 & 17 & 3.268 \\
3 & 1.000 & 8 & 2.130 & 13 & 2.854 & 18 & 3.356 \\
4 & 1.272 & 9 & 2.299 & 14 & 2.969 & 19 & 3.438 \\
5 & 1.519 & 10 & 2.455 & 15 & 3.075 & 20 & 3.515
\end{tabular}

scatter largely in the log scale. Substituting the extracted value of $\mu$ into the selfconsistent equations, we have the values of parameters: $q=0.431, \alpha_{0}=1.149$ and $X=0.307$. With these values, other quantities are determined, e.g., $\Delta \alpha=1.248$, $\alpha_{+}-\alpha_{0}=\alpha_{0}-\alpha_{-}=0.7211$ and $\omega_{n}^{\dagger}=0.557\left(\alpha^{\dagger}=1.005\right)$. We see an excellent agreement between the measured PDF of accelerations and the analytical formula of PDF derived by the present self-consistent multifractal analysis. The value of $\omega_{n}^{\dagger}$ tells us that the contribution from thermal fluctuation and/or measurement error is restricted to smaller values of $\omega_{n}$, i.e., less than about one half of its standard deviation. Then, the values of $\alpha$ responsible for the intermittency due to the scale invariance turn out to be smaller than $\alpha^{\dagger} \approx 1$. This is the range within the condition $\alpha<1.5$ in which the singularity appears in fluid particle accelerations.

The values of the scaling exponents $\zeta_{m}$, given by $(7)$, for $m=1, \ldots, 20$ are listed in table 1 for future convenience in comparison with experiments or other theories. Note that $\mu$ is related to $\zeta_{6}$ by the relation $\mu=2-\zeta_{6}$ within the present analysis. The flatness $F_{\mathrm{a}}^{(n)} \equiv\left\langle\left\langle\mathrm{a}_{n}^{4}\right\rangle\right\rangle /\left\langle\left\langle\mathrm{a}_{n}^{2}\right\rangle\right\rangle^{2}=\left\langle\left\langle\omega_{n}^{4}\right\rangle\right\rangle$ of the PDF of accelerations has the value $F_{\mathrm{a}}^{(n)}=57.8$ which is compatible with the value of the flatness $\sim 60$ reported in $[21,22]$ as it should be. It is quite attractive to see that the distance $r=\ell_{n}$ corresponding to the extracted value $n=16.4$ reduces to $r=0.821 \mu \mathrm{m}(r / \eta=0.0456)$, which is close to the value of the spatial resolution $0.5 \mu \mathrm{m}$ (1/40 of the Kolmogorov distance) of the measurement in $[21,22]$. Here, we used $\ell_{0}=0.071 \mathrm{~m}$ reported in $[21,22]$.

We derived, in this letter, the formula of the PDF of accelerations (8) within the approach of the multifractal analysis constructed by the present authors, and successfully analyzed the beautiful experiment conducted by Bodenschatz and coworkers $[21,22]$ in the Lagrangian frame. We expect that other data for the same system, in addition to the PDF of accelerations, will be provided in the near future, such as the intermittency exponent $\mu$, the scaling exponents $\zeta_{m}$, the PDF's of the velocity fluctuations or of the velocity derivatives. Then, we can cross-check the validity of the present analysis based on the formula (8). ${ }^{4}$ Note that the formula for the PDF

\footnotetext{
${ }^{4}$ We have checked, with the help of the DNS data reported by Gotoh et al. in [27], the accuracy of the value of the intermittency exponent extracted out of the measured PDF's. With the formula (7), we determined in [8] the value $\mu=0.240$ for the longitudinal velocity fluctuations by fitting, with the method of least squares, the ten data of the scaling exponents $\zeta_{m}(m=1,2, \cdots, 10)$ at $\mathrm{R}_{\lambda}=381$. On the other hand, we have extracted the value $\mu=0.237$ by the method of least
} 
of accelerations is different from the one for the PDF's of velocity fluctuations or of velocity derivatives. The empirical PDF, $\hat{\Lambda}_{\mathrm{emp}}(\omega)=C \exp \left\{-\omega^{2} /\left[\left(1+|\omega \beta / \sigma|^{\gamma}\right) \sigma^{2}\right]\right\}$ with $\beta=0.539, \gamma=1.588, \sigma=0.508$ and $C=0.786$ proposed in $[21,22]$ for the data at $R_{\lambda}=970$, gives a line very close to the one provided by the present PDF $\hat{\Lambda}^{(n)}(\omega)$ of $(8)$ for the region $|\omega|<30$ where the data of PDF exist. They deviate, however, for $|\omega|>30$, i.e., $\hat{\Lambda}_{\text {emp }}(\omega)<\hat{\Lambda}^{(n)}(\omega)$. The extraction of the PDF of the accelerations out of the DNS data obtained by Gotoh et al. [27] is one of the attractive investigations in order to check the validity of the present formula (8) derived by the unified approach providing various PDF's [5-9], since the accuracy of their DNS data for PDF's is very high up to the order of $10^{-10}$ [28].

\section{Acknowledgement}

The authors would like to thank Prof. C.Tsallis for his fruitful comments with encouragement. The authors are grateful to Prof. E.Bodenschatz for his kindness to show his data prior to publication.

\section{References}

1. Arimitsu T., Arimitsu N. // Phys. Rev. E, 2000, vol. 61, No. 3, p. 3237-3240.

2. Arimitsu T., Arimitsu N. // J. Phys. A: Math. Gen., 2000, vol. 33, p. L235-L241 [CORRIGENDUM: 2001, vol. 34, p. 673-674].

3. Arimitsu T., Arimitsu N. // Chaos, Solitons and Fractals, 2002, vol. 13, p. 479-489.

4. Arimitsu T., Arimitsu N. // Prog. Theor. Phys., 2001, vol. 105, No. 2, p. 355-360.

5. Arimitsu T., Arimitsu N. // Physica A, 2001, vol. 295, p. 177-194.

6. Arimitsu N., Arimitsu T. // J. Korean Phys. Soc., 2002, vol. 40, No. 6, p. 1032-1036.

7. Arimitsu T., Arimitsu N. // Physica A, 2002, vol. 305, p. 218-226.

8. Arimitsu T., Arimitsu N. // J. Phys.: Condens. Matter, 2002, vol. 14, p. 2237-2246.

9. Arimitsu N., Arimitsu T. // Europhys. Lett., 2002, vol. 60, No. 1, p. 60-65.

10. Rényi A. On measures of entropy and information. - In: Proc. 4th Berkeley Symp. Maths. Stat. Prob. Vol. 1, University of California Press, Berkeley, Los Angeles, 1961, p. 547.

11. Tsallis C. // J. Stat. Phys., 1988, vol. 52, p. 479-487.

12. Tsallis C. // Braz. J. Phys., 1999, vol. 29, No. 1, p. 1-35 (on the related recent progresses see at http://tsallis.cat.cbpf.br/biblio.htm).

13. Havrda J.H., Charvat F. // Kybernatica, 1967, vol. 3, p. 30-35.

14. Oboukhov A.M. // J. Fluid Mech., 1962, vol. 13, p. 77-81.

15. Kolmogorov A.N. // J. Fluid Mech., 1962, vol. 13, p. 82-85.

16. Yaglom A.M. // Sov. Phys. Dokl., 1966, vol. 11, p. 26-29.

17. Frisch U., Sulem P-L., Nelkin M. // J. Fluid Mech., 1978, vol. 87, p. 719-736.

18. Meneveau C., Sreenivasan K.R. // Phys. Rev. Lett., 1987, vol. 59, p. 1424-1427.

19. Meneveau C., Sreenivasan K.R. // Nucl. Phys. (Proc. Suppl.) B, 1987, vol. 2, p. 49-76.

20. Hosokawa I. // Phys. Rev. Lett., 1991, vol. 66, p. 1054-1057.

squares as the best fit of the theoretical formula for the PDF of velocity derivatives, derived in [9] by the multifractal analysis, to the corresponding PDF data. 
21. Porta A.La, Voth G.A., Crawford A.M., Alexander J., Bodenschatz E. // Nature, 2001, vol. 409, p. 1017-1019.

22. Voth G.A., Porta A.La, Crawford A.M., Alexander J., Bodenschatz E. // J. Fluid Mech., 2002, vol. 469, p. 121-160.

23. Kolmogorov A.N. // C.R. Acad. Sci. USSR, 1941, vol. 30, p. 301-305; p. 538-540.

24. Frisch U., Parisi G. Turbulence and Predictability in Geophysical Fluid Dynamics and Climate Dynamics. New York, North-Holland, 1985, p. 84.

25. Costa U.M.S., Lyra M.L., Plastino A.R., Tsallis C. // Phys. Rev. E, 1997, vol. 56, p. $245-250$.

26. Lyra M.L., Tsallis C. // Phys. Rev. Lett., 1998, vol. 80, p. 53-56.

27. Gotoh T., Fukayama D., Nakano T. // Phys. Fluids, 2002, vol. 14, p. 1065-1081.

28. Arimitsu T., Arimitsu N. Multifractal analysis of various probability density functions in turbulence. Preprint of arXiv.org e-Print archive, cond-mat/0301516, 2003, 8 p.

\title{
Аналіз прискорень при турбулентності на основі узагальненої статистики
}

\author{
Т.Аріміцу ${ }^{1,2}$, Н.Аріміцу ${ }^{2}$ \\ 1 Інститут фізики, університет м. Цукуба, Ібаракі 305-8571, Японія \\ 2 Національний університет м. Йокогама, Канагава 240-8501, Японія
}

Отримано 14 жовтня 2002 р.

За допомогою статистики, яка базується на узагальненій ентропії (ентропії Цалліса чи ентропії Рені) виведено аналітичний вираз для функції густини ймовірності прискорень при турбулентності. Виявлено, що дана функція пояснює результат, отриманий Боденшацом та ін. при вимірюванні прискорень частинок флюїду за умов повністю розвинутої турбулентності при $R_{\lambda}=970$.

Ключові слова: мультифрактальний аналіз, повністю розвинута турбулентність, функція густини ймовірності прискорень частинок флюїду, ентропія Рені, ентропія Цалліса

PACS: $47.27 .-i, 47.53 .+n, 47.52 .+j, 05.90 .+m$ 